\title{
Assessment Method of Emergency Preparedness System Vulnerability Based on the Complex Network Theory
}

\author{
Tianhan Jiang \\ China Academy of Safety Science and Technology, \\ Anquan Plaza, 32 Beiyuan Road, Chaoyang District, \\ Beijing100012,China. \\ E-mail:jiangth@chinasafety.ac.cn \\ Received 26 May 2012 \\ Accepted 16 October 2012
}

\begin{abstract}
A novel assessment method of emergency preparedness system vulnerability (EPSV) is established to evaluate the emergency system construction. The assessment index system and its complex network were constructed for EPSV. A simple weighted sum method was used to score the indices. And then the EPSV was calculated based on the Complex Network Theory. Finally, this model was applied to assessment EPSV of 3 regional governments. The results show that the emergency preparedness complex network can characterize the relationship between the components of an emergency preparedness system. This model can assessment the weak links and structural faults of regional emergency preparedness. The emergency preparedness complex network is rational, and the method is effective and feasible.
\end{abstract}

Keywords: emergency management, emergency incident, emergency preparedness, system vulnerability

\section{基于复杂网络的应急准备系统脆弱性评估方法}

\author{
江田汉 \\ 中国安全生产科学研究院, 北京 100012, 中国
}

\begin{abstract}
摘要：建立突发事件应急准备系统脆弱性评估方法, 为应急体系建设提供评估工具。选择突发事件应急准 备脆弱性评估指标, 建立应急准备系统脆弱性复杂网络, 采用简单加权求和方法计算指标得分, 最后基于 复杂网络计算突发事件应急准备系统脆弱性, 并将该评估指标和方法应用于 3 个实例。结果表明该应急准 备复杂网络较好地描述了突发事件应急准备体系各组成部分之间的关联和耦合关系。该方法可查找突发事 件应急准备能力的薄弱和不足之处, 评估应急准备系统结构性的缺陷。该应急准备系统脆弱性复杂网络较 合理、评估方法可行。
\end{abstract}

关键词: 应急管理, 突发事件, 应急准备, 系统脆弱性

1. 引言

应急准备过去被作为应急管理的四个阶段一减灾、 准备、响应、恢复的一个组成部分。“9-11” 事件 之后, 突发事件应急管理由以应急处置为主向应急 准备为核心转变 ${ }^{[1]}$ 。应急准备被定义为 “一系列事 先精心设计的关键任务和行动, 以建立、保持和改 进国内各类事件的预防、保护、响应、恢复所必须 的操作能力。应急准备是一个连续的过程, 包含各 级政府的努力, 政府、私人部门和非政府组织间的
协调行动, 以识别危险、确定脆弱性和识别所需要 的资源” ${ }^{[1]}$ 。国家应急准备的周期包括计划、组 织、装备和培训、演练、评估和改进等关键环节 ${ }^{[2,3]}$, 从而把应急准备提升为 “预防、保护、响应、 恢复”之外的基础性、全过程的行动。因此，应急 准备是指为有效应对突发事件，提高应急管理能力 而采取的各种措施与行动的总称, 包括意识、组 织、机制、预案、队伍、资源、培训演练等各种准 备 ${ }^{[4]}$ 。卡特里娜风䢐风造成严重后果主要原因之一是 应急准备不足和应急管理系统脆弱性 ${ }^{[5]}$ 。在应急准 
备体系中加强对脆弱性管理已成为美国国家政策 $[1,6,7]$ 。

脆弱性是面对灾害时唯一可以真正控制的因素 ${ }^{[8]}$ 。 不同领域对 “脆弱性” 概念的理解存在差异, 且由 于脆弱系统的复杂性, 脆弱性评价研究尚未成熟 [9]。目前, 脆弱性概念主要侧重于 “结果” 或 “脆 弱性的表现及其原因” ${ }^{[10]}$, 有风险-灾害 (RH）模 式、压力释放 (PAR) 模式、政治经济模式、基于区 域的综合脆弱性模式和恢复力模式等 5 种经典脆弱 性分析模型 ${ }^{[11]}$ 。脆弱性评价方法主要有综合指数 法、图层叠置法、脆弱性函数模型评价法、模糊物 元评价法和危险度分析法等 ${ }^{[10]}$ 。应急体系脆弱性相 关研究也逐渐展开, 如基于历史数据、指标体系和 实际调查的灾损率曲线的自然灾害脆弱性评估 ${ }^{[11]}$, 在应急管理人力资源 ${ }^{[12]}$ 、应急管理的法规及其相关 制度、个人、家庭、组织和社区等应对主体等在应 急体系中的作用 ${ }^{[13]}$ 、应急管理脆弱性来源及其发生 模型 ${ }^{[8]}$, 这些研究多涉及应急准备的若干方面 ${ }^{[12,13]}$ 或 侧重于脆弱性评估概念模型 ${ }^{[14,15]}$ 。应急管理脆弱性 的研究应得到重视 ${ }^{[16]}$, 脆弱性分析应成为应急预案 必不可少的组成部分 ${ }^{[17]}$ 。

笔者曾提出基于风险的应急准备脆弱性评估指标体 系和评估方法, 用于查找区域突发事件应急准备工 作中薄弱环节和不足之处 ${ }^{[18]}$ 。但该方法不能识别我 国应急准备体系结构性缺陷和制度化薄弱 ${ }^{[17]}$ 。文 中，考虑突发事件应急准备脆弱性要素之间的关联 度和耦合性, 基于复杂网络理论建立全风险的突发
事件应急准备系统脆弱性评估方法。

\section{2. 评估方法}

\section{1. 评估指标}

根据《突发事件应对法》、“一案三制” 的应急管 理体系框架以及城市重大事故应急能力评估指标体 系 $^{[18-20]}$, 经过多轮次的专家访谈, 选择由 11 个一级 指标、45 个二级定性指标构成的突发事件应急准备 脆弱性评估指标体系, 见表 1 。特别指出的是, 本 评估指标体系仅适用于省级地方人民政府，目的是 查找各地区应急准备工作中存在的问题和不足，持 改进各地区突发事件应急体系建设, 不断提高各级 人民政府突发事件应对能力。这是针对各类突发事 件应急准备脆弱性的共性问题的综合评估，不适用 于具体某类突发事件的评估。

\section{2. 计算方法}

\subsection{1. 应急准备脆弱性指标评分计算}

采用正面描述的方法对每个指标分为优、良、中、 差 4 个级别, 分别赋分值 $0 、 1 、 2$ 和 3 。

(1) 二级指标评分计算

假设有 $\mathrm{P}$ 个评估人员独立评分，则每个二级指标有 $\mathrm{P}$ 个分值。该二级指标评分按如下规则计算,

表 1 突发事件应急准备脆弱性评估指标

\begin{tabular}{|c|c|c|c|}
\hline 一级指标 & 二级指标 & 一级指标 & 二级指标 \\
\hline 1. 应急预案制订与管理 & $\begin{array}{l}1.1 \text { 应急预案制定小组 } \\
1.2 \text { 风险分析 } \\
1.3 \text { 应急能力评估 } \\
1.4 \text { 应急预案评审 } \\
1.5 \text { 预案的修订改进 } \\
1.6 \text { 应急预案体系 }\end{array}$ & 7. 应急恢复 & $\begin{array}{l}7.1 \text { 调查评估 } \\
7.2 \text { 恢复重建计划 } \\
7.3 \text { 总结报告 }\end{array}$ \\
\hline 2. 应急法制与制度化建设 & $\begin{array}{l}2.1 \text { 应急法制 } \\
2.2 \text { 应急制度 } \\
2.3 \text { 应急体系建设规划 }\end{array}$ & 8. 宣传、教育和培训 & $\begin{array}{l}8.1 \text { 应急管理培训 } \\
8.2 \text { 应急管理宣传 } \\
8.3 \text { 应急知识教育 }\end{array}$ \\
\hline 3. 应急组织体系 & $\begin{array}{l}3.1 \text { 领导机构 } \\
3.2 \text { 办事机构 } \\
3.3 \text { 工作机构 } \\
3.4 \text { 专家组 }\end{array}$ & 9. 应急演练 & $\begin{array}{l}9.1 \text { 演练规划 } \\
9.2 \text { 演练组织实施 } \\
9.3 \text { 演练总结评估与改进 }\end{array}$ \\
\hline 4. 风险评估体系建设 & $\begin{array}{l}4.1 \text { 重大危险源 } \\
4.2 \text { 危险区域 } \\
4.3 \text { 隐治理 } \\
4.4 \text { 综合减灾计划 } \\
4.5 \text { 风险评估 }\end{array}$ & 10. 应急基础保障 & $\begin{array}{l}10.1 \text { 应急指挥平台 } \\
10.2 \text { 应急物资 } \\
10.3 \text { 应急装备 } \\
10.4 \text { 应急资金 } \\
10.5 \text { 突发事件信息系统 } \\
10.6 \text { 应急通信 }\end{array}$ \\
\hline 5. 监测预警 & $\begin{array}{l}5.1 \text { 突发事件监测 } \\
5.2 \text { 突发事件预警 } \\
5.3 \text { 突发事件信息报告 }\end{array}$ & 11. 应急救援队伍 & $\begin{array}{l}11.1 \text { 军队武警 } \\
11.2 \text { 综合性应急救援队伍 } \\
11.3 \text { 专业性应急救援队伍 } \\
11.4 \text { 基层综合性应急救援队伍 } \\
11.5 \text { 志愿者队伍 }\end{array}$ \\
\hline 6. 应急指挥协调 & $\begin{array}{l}6.1 \text { 重大突发事件统一指挥 } \\
6.2 \text { 应急响应的部门协调 } \\
6.3 \text { 跨区的应急协调 } \\
6.4 \text { 应急救援人员的安全 }\end{array}$ & & \\
\hline
\end{tabular}




$$
S_{2}=\frac{N_{2}+2 N_{3}+3 N_{4}}{N_{1}+N_{2}+N_{3}+N_{4}}
$$

其中, $N_{1} 、 N_{2} 、 N_{3}$ 和 $N_{4}$ 分别为将指标评为优、良、 中、差的专家数, $P=N_{1}+N_{2}+N_{3}+N_{4}$ 。

(2) 一级指标评分计算

假设某一级指标分为 $k$ 个二级指标, 其得分记为 $S_{21}, S_{22}, \cdots \cdots, S_{2 k}$ ( $k$ 为整数), 则该一级指标的 评分 $\mathrm{S}_{1}$ 按如下规则计算:

$$
S_{1}=\sum_{i=1}^{k} W_{2 i} S_{2 i}
$$

式中, $W_{2 \mathrm{i}}$ 为二级指标权重, 若没有确定, 则 $W_{2 \mathrm{i}}$ 默认 为 $1 / k$ 。

\subsection{2. 应急准备系统脆弱性计算}

将各级各个评估指标等效为节点, 如果两指标之间 存在关联度和耦合关系，则在它们之间建立一条
按式（1）和式（2）计算各节点 $i$ 的初始脆弱性, 记为 $S_{i}$, 记节点 i 指向节点 $\mathrm{j}$ 的权重为 $W_{i j}$, 节点 $\mathrm{i}$ 的度为 $D_{i}$, 节点总数为 $N$, 则节点 $\mathrm{i}$ 脆弱性 $V_{i}$ 按 式 (3) 计算:

$$
V_{i}=\frac{S_{i}+\sum_{\mathrm{j}=1, \mathrm{j} \neq i}^{N} S_{\mathrm{j}} W_{j i}}{D_{i}}
$$

系统脆弱性 $V_{I}$ 按式 (4) 计算:

$$
V_{I}=\frac{\sum_{i=1}^{N}\left(S_{i}+\sum_{\mathrm{j}=1, \mathrm{j} \neq i}^{N} S_{\mathrm{j}} W_{j i}\right)}{\sum_{i=1}^{N} D_{i}}
$$

式中，符号意义同上。

文中各指标的权重均为默认值。

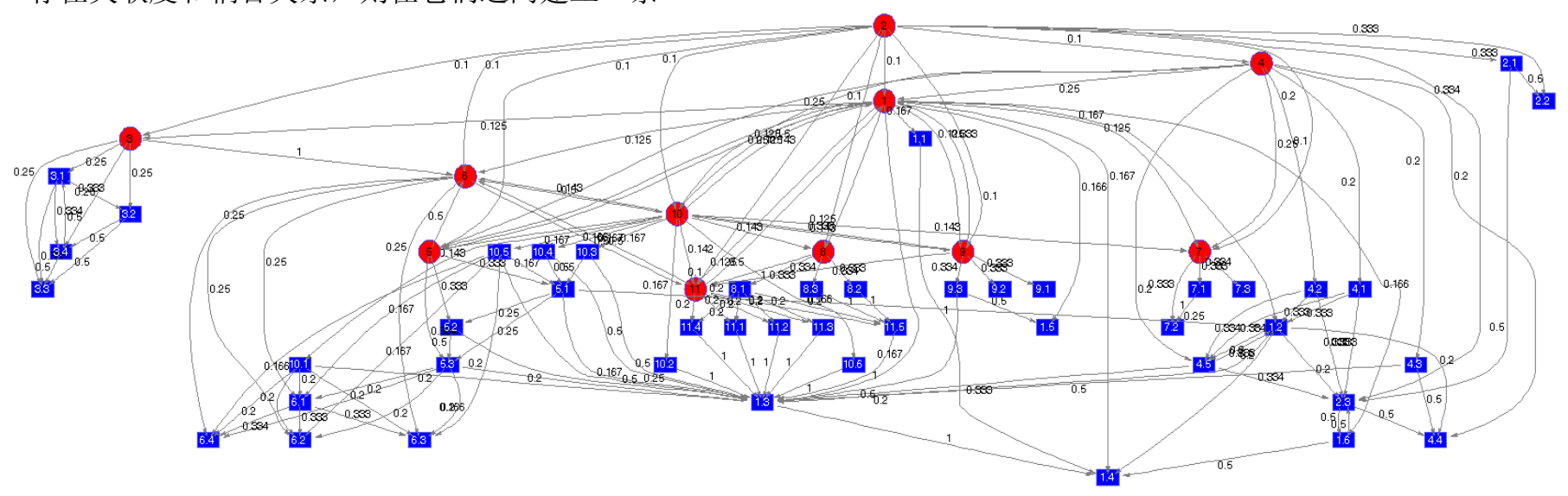

图 1 应急准备系统脆弱性网络

边，并赋予合适的权重，并建立如下规则:

(1) 一级指标必与其二级指标有单向边相连;

（2）一级指标可与其他二级指标无单向边相连;

(3) 二级指标与一级指标无单向边相连;

（4）二级指标可与其他二级指标有单向边相连;

（5）一级指标与其二级指标之间边的权重赋予平均 值;

（6）各指标出度的权重赋予平均值, 如果出度为 1 , 则权重为 1 ;

对评估指标进行因果分析 ${ }^{[21]}$ ，结合专家的先验知 识, 建立应急准备系统脆弱性网络如图 1。其中, 红色节点为一级指标, 蓝色节点为二级指标。

\section{3. 应用实例}

将评估模型应用于 $A 、 B 、 C$ 三地，以检验该评估指 标体系和评估方法, 其评估过程如下: (1) 组织 $A$ 政管委、安监局、消防支队、民政局、交通支队、 公安局、电力公司、地震局、水务局、气象局、卫 生局等 12 个部门推荐的 15 位专家组成评估小组, 结合 $A$ 的实际情况, 对 $A$ 应急准备能力进行评估;

（2）组织 $B$ 政府办公室、安全生产监督管理局、消 防处、民政局、交通管理处、治安支队等部门的 9 位专家, 结合 $B$ 的实际情况, 对 $B$ 应急准备能力进 行实际评估; (3) 组织 $C$ 应急联动中心、安全生产 监督管理局、公安局、交警支队、地震局、民政 
局、卫生局、某公司等 11 位专家组成评估小组, 结 合 $C$ 的实际情况, 对 $C$ 应急准备能力进行客观评 估; 上述专家评分均在显著性水平 $a=0.05$ 下通过 了 kappa 一致性检验, 具体方法见文献 [22]。然后

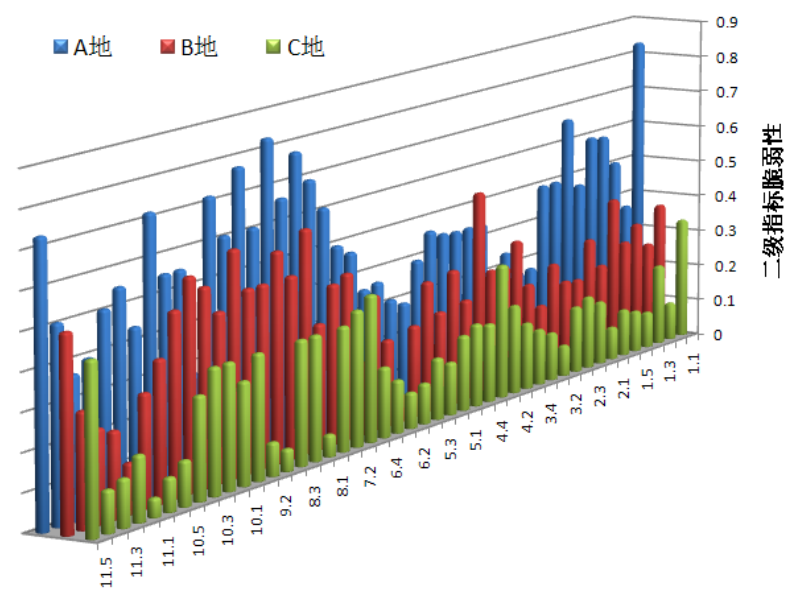

图 2 应急准备二级指标脆弱性

将应急准备能力评分转换为应急准备脆弱性得分, 并作归一化处理, 再分别计算各节点的脆弱性和系 统脆弱性。

\section{1. 二级指标脆弱性分析}

分析具体的二级指标可以详细地掌握突发事件应急 准备系统脆弱性突出之处。按式 (1) 计算 A、B、 C 三地应急准备一级指标的脆弱性, 结果见图 2。A 地应急指挥平台建设情况, B 地的重大突发事件统 一指挥和军队武警协同情况较好, 而 $\mathrm{C}$ 地志愿者队

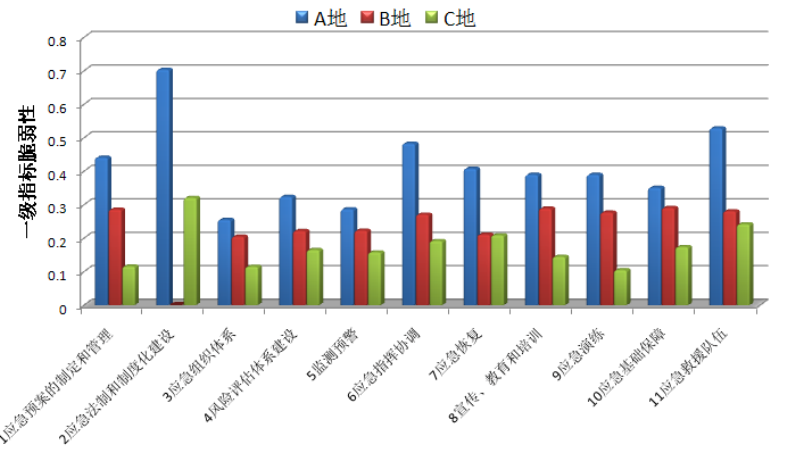

图 3 应急准备一级指标脆弱性

伍建设较差, 但应急预案制定、评审与修订改进工 作做得较好, 应急管理培训和演练规划做得最好。

\section{2. 一级指标脆弱性分析}

按式 (2) 算 A、B、C 三地应急准备一级指标的脆弱 性, 结果见图 3。

总体而言, A 地的 11 项一级指标脆弱性最大, C 地 最小， B 地介于两者之间。 A、B、C 三地在应急预案 制定与管理、应急法制和制度化建设、应急组织体 系、风险评估体系建设等方面建设得较好, 特别是 在应急组织体系、风险评估体系建设等方面。在监 测预警、应急指挥协调、应急恢复、宣传、教育和 培训、应急演练、应急救援队伍等方面的建设较 差。

\section{3. 系统脆弱性分析}

按式 (3) 和式 (4) 分别计算 A、B、C 三地突发事 件应急准备系统脆弱性, 结果见图 4。A 地应急准备 系统脆弱性最大, $\mathrm{B}$ 地次之, $\mathrm{C}$ 地最小。由于 $A$ 地在

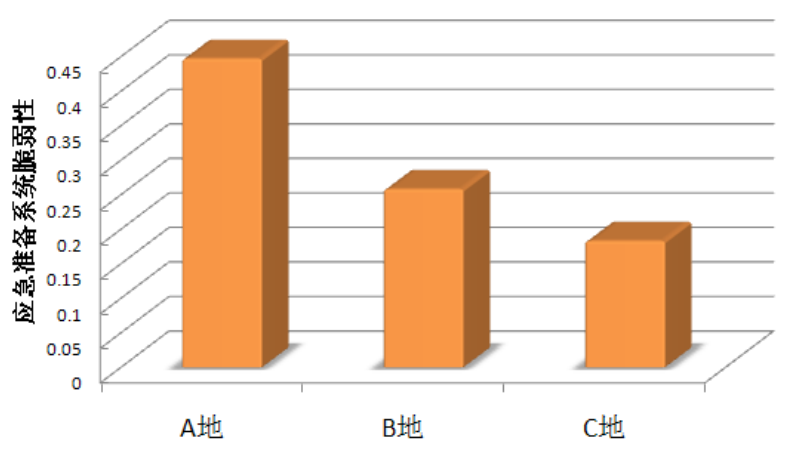

图 4 应急准备系统脆弱性

监测预警、应急指挥协调、应急恢复、应急演练等 方面最显薄弱与不足, 突发事件应急准备脆弱性的 多种影响因素在复杂的关联和耦合作用下, A 地应 急准备系统脆弱性最大, 反映出该地突发事件应急 准备系统脆弱性的总体情况。

关于应急准备体系建设, 笔者实地调研了 $\mathrm{A}$ 地应急 管理办公室、城市管理监督指挥中心、部分专项应 急指挥部和街乡镇基层组织, 发现各部门与街乡镇 应急能力建设不平衡, 布局不合理, 出现能力缺失 或者重复建设等现象, 如此必将造成突发事件应急 准备体系结构性的脆弱, 北京 “ 6 - 23 ” 特大暴雨发 生时 A 地应急抢险救援效果较差就是例证。

\section{4. 结论}

(1) 该方法较好地解决了突发事件应急准备系统脆 弱性评估问题, 可为突发事件应急体系建设提供参 考 
（2）。该应急准备复杂网络较好地描述了应急准备 不同组成部分、不同层级之间的相互作用相互影响 的关系, 能够从宏观和微观两个角度发现突发事件 应急准备能力的薄弱和不足之处。

应该指出的是该方法在指标以及指标间关联度和耦 合性的权重设置方面还待进一步完善。

\section{致谢}

本文受国家自然科学基金重大研究计划项目

（90924303、91024031）资助。

中国安全生产科学研究院刘铁民、李湖生、邓云 峰、姜传胜、王建光、王晶晶等同志参与了评估指 标体系与方法的讨论, 并提出了建设性的建议。

\section{参考文献}

[1] U.S.Department of Homeland Security.The national preparedness guidelines[R].[EB/OL].(2007-09-13)[201 0-04-05].http://www.dhs.gov/xlibrary/assets/National_ Preparedness \linebreak_Guidelines.pdf.

[2] U.S. Department of Homeland Security.The federal preparedness report[R].[EB/OL].(2009-01-26)[2010-04 -05].http://www.fas.org/irp/agency/dhs/fema/prep.pdf.

[3] U.S. Department of Homeland Security.National response framework[R].[EB/OL].(2008-01)[2010-04-0 5].http://www.fema.gov/pdf/emergency/nrf/nrf-core. pdf.

[4] Li Husheng,Liu Tiemin.The research progresses and key scientific problems on emergency preparedness system [J].Journal of Safety Science and Technology, 2009,5(6):5-10.

李湖生.刘铁民.突发事件应急准备体系研究进展及 关键科学问题 [J]. 中国安全生产科学技术,2009,5 (6):5-10.

[5] The White House.The Federal Response to Hurricane Katrina: Lessons learned[R].[EB/OL].(2006-02-23) [2012-05-22].http://georgewbush-whitehouse.archives. gov/reports/katrina-lessons-learned/.

[6] FEMA.Comprehensive Preparedness Guide(CPG) 101Developing and Maintaining Emergency Operations Plans.[EB/OL].(2010-11)[2012-05-22].http://www. fema.gov/pdf/about/divisions/npd/CPG_101_V2.pdf

[7] U.S. Department of Homeland Security.The 2009 National Infrastructure Protection Plan[R].[EB/OL]. (2009)[2012-05-22].http://www.dhs.gov/xlibrary/assets /NIPP_Plan.pdf.

[8] McEntire D A.The Status of Emergency Management Theory-Issues,Barriers, and Recommendations for Improved Scholarship[C].(2004-06-08)[2012-05-22]. http://www.training.fema.gov/emiweb/downloads/Davi d\%20McEntire\%20-\%20\%20Status\%20of\%20 Emergency \%20Management\%20Theory.pdf.

[9] Rygel L,O'sullivan D,Yarnal B.A method for constructing a social vulnerability index:an application to hurricane storm surges in a developed country[J].
Mitigation and Adaptation Strategies for Global Change, 2006,11(3):741-764.

[10] Li He,Zhang Pingyu,Cheng Yeqing.Concepts and Assessment Methods of Vulnerability[J].Progress in Geography,2008,27(2):18-25.

李鹤,张平宇, 程叶青.脆弱性的概念及其评价方法 [J]. 地理科学进展,2008,27(2):18-25.

[11] Shi Yong,Xu Shiyuan,Shi Chun,Sun ali,Zhao Qing liang.Progress in research on vulnerability of natural disasters[J]. Journal of Natural Disasters,2011(2):131137.

石勇,许世远,石纯,孙阿丽,赵庆良.自然灾害脆弱性研 究进展[J].自然灾害学报,2011,20(2):131-137.

[12] O'Brien G.UK Emergency preparedness:a step in the right direction?[J].Journal of International Affairs,2006, 59(2):63-86.

[13] Comfort L K.Rethinking security:organizational fragility in extreme events[J].Public Administration Review,2002, 62(S1):98-107.

[14] Liu Tiemin.Recognition of disaster causes-study of the vulnerability[J].Journal of Safety Science and Technology,2010,6(5):5-10. 刘铁民.事故灾难成因再认识——脆弱性研究 [J].中 国安全生产科学技术,2010,6(5):5-10.

[15] Shi Peijun.Theory on disaster science and disaster dynamics[J].Journal of Natural Disasters,2002,11(3):19.

史培军.三论灾害研究的理论与实践 $[\mathrm{J}]$. 自然灾害学 报,2002,11(3):1-9.

[16] Li Husheng,Jiang Chuansheng,Liu Tiemin.The key scientific problems and research progress of major crisis emergencies response[J].Journal of Safety Science and Technology,2008,4(5):13-18.

李湖生,姜传胜,刘铁民.重大危机事件应急关键科学 问题及其研究进展 [J].中国安全生产科学技术,2008, 4(5):13-18.

[17] Liu Tiemin.The significance of emergency preparedness highlighted by Yushu earthquake once again[J].Journal of Safety Science and Technology, 2010,6(2):5-7.

刘铁民.玉树地震灾害再次凸显应急准备重要性 [J] 中国安全生产科学技术,2010,6(2):5-7.

[18] Jiang Tianhan,Deng Yunfeng,Li Husheng,Liu Tiemin,Jiang Chuansheng,Wang Jingjing.Assessment Method of Regional Emergency Preparedness Vulnerability[J].Journal of Northeastern University (Natural Science),2011,32(S2): 273-278.

江田汉,邓云峰,李湖生,刘铁民,姜传胜,王晶晶.区域 突发事件应急准备脆弱性评估方法[J].东北大学学报 (自然科学版),2011,32(S2):273-278.

[19] Deng Yunfeng,Zheng Shuangzhong,Liu Gongzhi,Liu Tiemin.Study on city emergency capability assessment system[J].Journal of Safety Science and Technology, 2005,1(6):33-36.

邓云峰,郑双忠,刘功智,等.城市应急能力评估体系研 究[J].中国安全生产科学技术,2005,1(6):33-36. 
[20] Deng Yunfeng,Zheng Shuangzhong,Capability assessment for urban emergency incident readiness[J]. Journal of Safety Science and Technology,2006,2(2):9-

13.

邓云峰, 郑双忠.城市突发公共事件应急能力评估一 一以南方某市为例 [J].中国安全生产科学技术,2006,2 (2):9-13.

[21] Zheng Shuangzhong,Deng Yunfeng,Jiang Tianhan. Kappa Analysis on Assessment of Urban Emergency Capability[J]. China Safety Science Journal,2006,16(2): 69-72.

郑双忠, 邓云峰, 江田汉. 城市应急能力评估体系 Kappa 分析[J].中国安全科学学报,2006,16(2):69-72. 